\title{
A Cross-Sectional Study of Knowledge, Attitude, and Practice Towards Face Mask Use Amid the COVID-19 Pandemic Amongst University Students in Vietnam
}

\author{
Minh Cuong Duong ${ }^{1}\left[\right.$ D Hong Trang Nguyen ${ }^{2} \cdot$ Bich Thuy Duong $^{3}$
}

Accepted: 18 March 2021 / Published online: 27 March 2021

(c) Crown 2021

\begin{abstract}
Although Vietnam has been successful in the fight against the first wave of COVID-19, domestic outbreaks have continued to occur. Given university students constitute a significant proportion of the population in Vietnam, to improve the prevention and control of COVID-19 in Vietnam and comparable countries, we examined their knowledge, attitude, and practice (KAP) towards face mask use which is an important preventive measure and the association between the levels of COVID-19 knowledge and KAP towards face mask use. A cross-sectional survey was conducted on all students studying at Phenikaa University, Hanoi, using a self-developed questionnaire consisted of 42 questions regarding KAP towards face mask use as well as reuse of cloth mask, and COVID-19 knowledge. Among 728 participants, 40.9\% (298/728) were male, 63.2\% (460/728) were from health-related faculties, 46\% (335/728) lived in urban areas, and 51.9\% (378) had good levels of COVID-19 knowledge. Regarding face mask, most (89.7\%, 653/728) participants had good knowledge scores, $72.8 \%$ (530/728) had positive attitudes, and 76.5\% (557/728) had good practice scores. The most common types of masks used were surgical mask $(57.6 \%, 419 / 728)$, followed by non-antibacterial cloth mask $(23.1 \%, 168 / 728)$. Among 168 participants using non-antibacterial cloth mask, $43.5 \%$ reused masks (73/168), of whom 6.8\% (5/73) did not wash their masks at least daily. Face mask use knowledge was statistically associated with COVID-19 knowledge $(\mathrm{P}<0.001)$. Face mask use attitudes were statistically associated with education levels $(\mathrm{P}=0.02)$, and COVID-19 knowledge $(\mathrm{P}<0.001)$. Practice of face mask use was statistically associated with gender $(\mathrm{P}=0.03)$, geographical distribution $(\mathrm{P}=0.04)$, academic majors $(\mathrm{P}=0.02)$, and COVID-19 knowledge $(\mathrm{P}=0.01)$. The levels of KAP towards face mask use among Vietnamese university students are high. It is important to continue to enforce the mass masking policy, together with the ongoing community education with an emphasis on the route of COVID-19 transmission and how correct face mask use can stop the spread. To ensure the effectiveness of face masks, there is an urgent need to educate the community regarding the procedures for cloth masks re-use.
\end{abstract}

Keywords KAP $\cdot$ Vietnam $\cdot$ University students $\cdot$ Face mask

\section{Introduction}

An outbreak of the novel coronavirus infectious disease 2019 (COVID-19) caused by the severe acute respiratory syndrome, coronavirus 2 (SARS-CoV-2) has been reported in Wuhan, China since December 2019, and has become a pandemic [1,2]. Given Vietnam shares a long border with

Minh Cuong Duong and Hong Trang Nguyen have contributed equally to the work.

Minh Cuong Duong

minh.duong@unsw.edu.au

Extended author information available on the last page of the article
China, Vietnam reported its first confirmed Vietnamese case soon on 23rd January 2020 [3]. However, the infection rate remains low at 28 cases per 10 million people with some fluctuations due to domestic outbreaks of COVID-19 [3, 4]. As of 17 Feb 2021, according to the Vietnam Ministry of Health, a total of 2311 confirmed cases were reported, including $1573(68.1 \%)$ recovered, and 35 (1.5\%) related deaths [4].

Vietnam has applied several prevention and control measures since an early stage of the pandemic $[3,5]$. These measures include restrictions on international flights, movement restrictions, contact tracing, quarantine and social distancing, governance (e.g., socio-economic supports), lockdown, and increasing public health awareness through public health 
education campaign [3]. Notably face mask use has been mandatory in public places, hospitals, and schools $[3,4$, 6], with a VND 300,000 (US\$13) fine for non-compliance [7]. Compared with most countries worldwide, Vietnam has been successful in controlling COVID-19 [3, 6], but domestic outbreaks of COVID-19 continue to occur with the most recent in February 2021 that has led the government lock down some large cities [4]. Given the ongoing pandemic, these domestic outbreaks were not unexpected, but it raises the need to strengthen the community adherence to preventive measures including face mask use which is strongly affected by the community knowledge, attitudes, and practices (KAP) [2, 8]. In Vietnam and some other Asian countries such as China, Japan, and Korea, it is a common practice to wear face masks in public due to health and cultural reasons $[7,9,10]$. Therefore, the implementation of compulsory mask wearing may be less challenging that in other countries [3]. However, the actual KAP towards mass masking practice and potential associated conflicts remain unknown, particularly among the young adults - the major population in Vietnam [11]. Indeed, the KAP plays an integral role in determining the community readiness to accept the compulsory mass masking practice which is a behavioral change measure from health authorities [12]. KAP studies also provide baseline information to guide intervention to change misconceptions about compulsory face mask use amid the COVID-19 pandemic and to address potential conflicts that may arise. To prevent future domestic outbreaks and guarantee the final success in the fight against COVID19 in Vietnam and comparable countries, our study aimed to provide data to inform future efforts focusing on increasing community adherence to mask-wearing requirements by examining the KAP towards face mask use among university students in Vietnam during the COVID-19 pandemic and the association between the levels of COVID-19 knowledge and KAP towards face mask use.

\section{Methods}

\section{Study Design and Setting}

A cross-sectional study was conducted between 2nd July 2020 and 2nd August 2020 at Phenikaa University—a large multidisciplinary university in Hanoi, Vietnam. All 902 students including 576 students from the health-related faculties (Faculty of Nursing and Faculty of Pharmacy) and 326 students from non-health related faculties were invited to participate in the study. Written informed consent was obtained from all study participants. The study was approved by the University Ethics Committee (reference No. 09/ TT-KDD).
To the best of our knowledge, face masks with ear loops are used in Vietnam, while face mask with ties or bands are not available. At the time the study was conducted there was no outbreak of COVID-19 in Vietnam.

The questionnaire included demographic information, 17 questions for KAP towards face mask use, five questions for the reuse of cloth mask, and 20 questions for COVID-19 knowledge. Seventeen questions for KAP towards face mask use were classified into three groups including knowledge (five questions), attitude (four questions), and practice (eight questions). These questions were based on the recommendations of the WHO and Vietnam Ministry of Health [13, 14]. The types of the questions included (1) single-choice questions in which, participants could only choose one response from a predetermined list, and (2) array questions in which, participants could either chose 'yes', 'no' or 'not sure' for their responses. Each correct answer was awarded one score and a selection of "not sure" was awarded a zero score. There were also Likert scale questions with four options corresponding to the levels of agreement/disagreement with the provided statements and to a score range of zero to three.

The knowledge questions included two single-choice and three array questions and thus, the total highest score for knowledge was 5 . The attitude questions included two single-choice, one array, and one Likert scale questions and thus, the total highest score for attitude was 6 . The practice questions included six single-choice, and two Likert scale questions and thus, the total highest score for practice was 12. An additional question was also included to ask for the most used face mask of study participants. For those who used non-antibacterial cloth masks, further three questions were asked regarding the reuse of cloth mask, the methods of re-use employed. Other 20 COVID-19 related single-choice and array questions were designed to assess the levels of COVID-19 knowledge including risk factors, routes of transmission, signs and symptoms, and the effectiveness of the preventive measures. Demographic information included age, gender, geographical distribution (urban and rural areas), level of education (first year, second year, third year, and the fourth year-final year), academic majors (health related majors and non-health related majors), and living conditions (living alone, with the seniors (grandparents, parents, uncles, and/or aunties), with siblings and/or cousins, and with friends). To ensure that the study participants fully understood the questionnaire, the questionnaire was in Vietnamese (a translated questionnaire is in Online Appendix 1). Also, assistance with the questionnaire was provided by the researcher (HTN).

\section{Statistical Analysis}

Data were managed and analyzed using the Statistical Package for the Social Sciences (SPSS) version 26 (IBM). 
Continuous variables were displayed as mean \pm one standard deviation (SD), and range. Categorical variables were presented as an absolute count and percentage. In this study, for the identification of the participants' level of each of the three components of KAP, the cut-off value was calculated and was equal to the mean score achieved by all study participants in each component minus one SD. Given the individual's score was a whole number, if the cut-off value was a decimal number, a good level of each of the three components of KAP was defined as an individual's score higher than or equal to nearest whole number higher than the cut-off value, while an individual's score lower than the nearest whole number higher than the cut-off value indicated a poor level. If the cut-off value was a whole number, a good level was defined as an individual's score higher than the cut-off value, while an individual's score lower than or equal to the cut-off value indicated a poor level. Chi-square test and Chi-squared test for trend were used to calculate significance levels for categorical data. T-test was utilized for the comparison of continuous data. The significance was set at $\mathrm{P} \leq 0.05$.

\section{Results}

\section{Baseline Characteristics of Study Participants}

Among all 902 invited students, 728 (81\%) participated in the study with a mean age of $20.8 \pm 1.4$ years. Of these 728 participants, 298 (40.9\%) were male, 460 (63.2\%) were from health-related faculties, $335(46 \%)$ lived in urban areas, and $132(18.1 \%)$ lived alone (Table 1$)$.

\section{Knowledge, Attitudes, and Practices Related to Face Mask Use and Levels of COVID-19 Knowledge Among 728 Study Participants}

The mean face mask use knowledge score was $2.3 \pm 0.8$ and $89.7 \%$ (653/828) of study participants had a good knowledge score (Table 2). The mean face mask use attitude score was $4.8 \pm 0.8$ and $72.8 \%$ (530/728) of participants had positive attitudes towards face mask use. The mean face mask use practice score was $8.5 \pm 1.5$ and $76.5 \%$ (557/728) of participants had a good practice score. More than half $(51.9 \%$, 378/728) of study participants had good levels of COVID-19 knowledge. The detail of the participants' response to each KAPs questions was outlined in the Online Appendix 2.

\section{The Most Commonly Face Mask Used Among Study Participants and Reuse of Cloth Masks}

More than half $(57.6 \%, 419 / 728)$ reported that a surgical mask was their most common face mask, followed by
Table 1 Demographic characteristics, living conditions, academic major, and level of education of 728 university students

\begin{tabular}{ll}
\hline Characteristics & Summary statistics* \\
\hline Age (years) & $20.8 \pm 1.4(19-34)$ \\
Male & $298(40.9)$ \\
Living in urban areas & $335(46)$ \\
Living conditions & \\
Alone & $132(18.1)$ \\
With the seniors (grandparents, parents, & $274(37.6)$ \\
$\quad$ uncles, and/or aunties) & $124(17.1)$ \\
With siblings and/or cousins & $198(27.2)$ \\
With friends & $460(63.2)$ \\
Health related majors & \\
Level of education & $113(15.5)$ \\
1st year & $302(41.5)$ \\
2nd year & $186(25.6)$ \\
3rd year & $127(17.4)$ \\
4th year (final year) &
\end{tabular}

*Mean \pm SD (min-max) for continuous variables and $\mathrm{n}(\%)$ for categorical variables

Table 2 Knowledge, attitudes, and practices related to face mask use and levels of COVID-19 knowledge among 728 university students

\begin{tabular}{ll}
\hline Items & Statistics* \\
\hline $\begin{array}{l}\text { Knowledge, attitudes, and practices related to face } \\
\text { mask use }\end{array}$ \\
Knowledge score \\
Knowledge score group \\
$\quad 2.3 \pm 0.8(0-5)$ \\
Poor & $75(10.3)$ \\
Good & $653(89.7)$ \\
Attitude score & $4.8 \pm 0.8(1-6)$ \\
Attitude score group & $198(27.2)$ \\
$\quad$ Negative & $530(72.8)$ \\
Positive & $8.5 \pm 1.5(2-12)$ \\
Practice score & \\
Practice score group & $171(23.5)$ \\
Poor & $557(76.5)$ \\
$\quad$ Good & \\
Levels of COVID-19 knowledge & $378(51.9)$ \\
Good & $350(48.1)$ \\
Poor &
\end{tabular}

$*$ Mean \pm SD (min-max) for continuous variables, while $\mathrm{n}(\%)$ for categorical variables

non-antibacterial cloth mask $(23.1 \%, 168 / 728)$, antibacterial cloth mask $(17.2 \%, 125 / 728)$, N95 respirator and other comparable face masks $(1.8 \%, 13 / 728)$, and activated carbon filter face masks $(0.4,3 / 728)$ (data not shown). Among 168 participants using a non-antibacterial cloth mask, $73(43.5 \%)$ reported to reuse the face mask, of whom $28.8 \%(21 / 73)$ washed their masks after each use, 64.4\% (47/73) after every 
day of use, $5.5 \%$ (4/73) after some days of use but less than a week, and $1.3 \%$ (1/73) after every week of use (Table 3). The most common reason why to reuse a face mask was to follow the manufacturer's recommendation.

\section{Factors Affecting Knowledge, Attitudes, and Practices Related to Face Mask Use}

There was a significant association between face mask use knowledge and COVID-19 knowledge $(\mathrm{P}<0.001)$ (Table 4). There was a significant association between face mask use attitudes and level of education $(\mathrm{P}=0.02)$ as well as COVID19 knowledge $(\mathrm{P}<0.001)$. There was a significant association between the practice of face mask use and male gender $(\mathrm{P}=0.03)$, geographical distribution $(\mathrm{P}=0.04)$, academic major $(\mathrm{P}=0.02)$, and COVID-19 knowledge $(\mathrm{P}=0.01)$.

\section{Discussion and Conclusion}

\section{Discussion}

Several countries worldwide continue to report high numbers of infected cases and deaths due to COVID-19 [15]. Although the infection and fatality rates remain low, Vietnam has experienced continuous domestic outbreaks of COVID-19 [4]. Given the increasing evidence of efficacy of COVID-19 preventive measures [9, 10, 16-20], ensuring community compliance with these preventive measures remains of vital importance [12]. Vietnam has implemented a mass masking policy together with other preventive measures since early 2020 [5, 7, 18]. However, a very few studies had been conducted to examine the adherence to this

Table 3 Characteristics of face mask reuse among 168 university students using non-antibacterial cloth mask

\begin{tabular}{ll}
\hline Items & $\mathrm{n}(\%)$ \\
\hline Reusing mask & $73(43.5)$ \\
How to reuse mask $(\mathrm{n}=73)$ & $21(28.8)$ \\
Wash after each use & $47(64.4)$ \\
Wash after every day of use & $4(5.5)$ \\
Wash after some days of use but less than a week & $1(1.3)$ \\
Wash after every week of use & 0 \\
Wash after more than seven days of use & 0 \\
Wash when it is visibly dirty or smells & 0 \\
Continue to use and there is no need to wash & \\
Why to reuse face mask (n=73) & $25(34.3)$ \\
The mask is still clean (there is no visible dirt) & $41(56.2)$ \\
Reusing the face mask in accordance with the manufac- & \\
turer's recommendation & $7(9.6)$ \\
Saving money &
\end{tabular}

policy in the general population [6], and there are no studies assessing the KAP towards face mask use among university students in Vietnam [11]. To the best of our knowledge, this is the first study in Vietnam and amongst very few studies in the world examining this issue in university students $[4,15]$. We believe it provides important scientific evidence regarding the implementation of the mass masking policy among university students who constitute a significant proportion of the population in Vietnam.

The demographic characteristics of our study population demonstrated a predominantly female population which is in line with other studies conducted in the general community in Vietnam [21, 22]. We found that $89.7 \%$ of our study participants had a good knowledge score, $72.8 \%$ had positive attitudes, and $76.5 \%$ had a good practice score regarding face mask use. On the one hand, our findings are better than the findings of a study conducted in Saudi Arabia where 44\% of the study population had little knowledge related to mask wearing and infection prevention. This may be because the Saudi Arabian study was conducted in March 2020 -during the early stage of the pandemic, while our study was conducted in July 2020 when several community education program had been implemented in Vietnam [18]. On the other hand, our findings are not as good as the findings of a study conducted in Hubei, China in which $98.0 \%$ followed the local mass masking policy [23]. This may be because the Chinese study was conducted in January 2020, the week immediately after the lockdown of Hubei Province, when the control and preventive measures were strictly enforced in this Province. University students are at risk of transmitting COVID-19 to other people provided that they are active population who can gather in groups at university and have high levels of social interaction. To prevent future outbreaks and ensure the success in fighting COVID-19 in Vietnam, our findings highlight the need to continue to enforce the mass masking policy, as a means of preventing the spread of the virus.

The two most common types of face mask used by our participants were surgical mask (57.6\%) and non-antibacterial cloth mask (23.1\%). To the best of our knowledge, these are also the two most popular face masks known by Vietnamese people prior to COVID-19. Before the pandemic, surgical masks were used by healthcare workers and, a lesser extent, the general community, while non-antibacterial cloth masks were used by the general community to protect their skin from ultraviolet light and air pollution [7]. Regarding cloth masks, in addition to members of the community, cloth masks were also widely used by healthcare workers in Asia including Vietnam [24]. In the context of COVID-19 pandemic, shortages of personal protective equipment have further led to widespread use of cloth mask [24]. Most people (56.2\%) who reused non-antibacterial cloth mask reported that they followed the manufacturer's recommendation and 
Table 4 The difference in knowledge, attitudes and practices related to face mask use among 728 university students

\begin{tabular}{|c|c|c|c|c|c|c|c|c|c|}
\hline \multirow[t]{2}{*}{ Characteristics } & \multicolumn{2}{|l|}{ Knowledge } & \multirow{2}{*}{$\begin{array}{l}\mathrm{p} \\
\text { OR }(95 \% \mathrm{CI})\end{array}$} & \multicolumn{2}{|l|}{ Attitudes } & \multirow{2}{*}{$\begin{array}{l}\mathrm{p} \\
\text { OR }(95 \% \mathrm{CI})\end{array}$} & \multicolumn{2}{|l|}{ Practices } & \multirow{2}{*}{$\begin{array}{l}\mathrm{p} \\
\mathrm{OR}(95 \% \mathrm{CI})\end{array}$} \\
\hline & $\begin{array}{l}\text { Poor } \\
(n=75)\end{array}$ & $\begin{array}{l}\text { Good } \\
(\mathrm{n}=653)\end{array}$ & & $\begin{array}{l}\text { Negative } \\
(\mathrm{n}=198)\end{array}$ & $\begin{array}{l}\text { Positive } \\
(\mathrm{n}=530)\end{array}$ & & $\begin{array}{l}\text { Poor } \\
(n=171)\end{array}$ & $\begin{array}{l}\text { Good } \\
(\mathrm{n}=557)\end{array}$ & \\
\hline $\begin{array}{l}\text { Age (years) } \\
(\text { mean } \pm \mathrm{SD})\end{array}$ & $20.8 \pm 1.36$ & $20.8 \pm 1.43$ & $0.58^{\mathrm{a}}$ & $20.7 \pm 1.4$ & $20.8 \pm 1.4$ & $0.8^{\mathrm{a}}$ & $21 \pm 1.5$ & $20.7 \pm 1.4$ & $0.07^{\mathrm{a}}$ \\
\hline Male (n, \%) & $32(42.7)$ & $266(40.7)$ & $0.8^{\mathrm{b}}$ & 89 (44.9) & 209 (39.4) & $0.2^{\mathrm{b}}$ & $83(48.5)$ & 215 (38.6) & $\begin{array}{l}0.03^{\mathrm{b}} \\
1.5(1.06-2.12)\end{array}$ \\
\hline $\begin{array}{l}\text { Living in urban } \\
\text { areas }(\mathrm{n}, \%)\end{array}$ & $37(49.3)$ & $298(45.6)$ & $0.54^{\mathrm{b}}$ & $92(46.5)$ & $243(45.8)$ & $0.93^{\mathrm{b}}$ & $67(39.2)$ & $268(48.1)$ & $\begin{array}{l}0.04^{\mathrm{b}} \\
0.69(0.49-0.98)\end{array}$ \\
\hline $\begin{array}{l}\text { Living condi- } \\
\text { tions (n, \%) }\end{array}$ & & & $0.13^{\mathrm{c}}$ & & & $0.06^{\mathrm{c}}$ & & & $0.09^{c}$ \\
\hline Alone & $8(10.7)$ & $124(19)$ & & $43(21.7)$ & 89 (16.8) & & $32(18.7)$ & $100(18)$ & \\
\hline $\begin{array}{l}\text { With the } \\
\text { seniors* }\end{array}$ & $25(33.3)$ & $249(38.1)$ & & $65(32.8)$ & 209 (39.4) & & $74(43.3)$ & $200(35.9)$ & \\
\hline $\begin{array}{l}\text { With siblings } \\
\text { and/or } \\
\text { cousins }\end{array}$ & $16(21.3)$ & $108(16.5)$ & & $42(21.2)$ & $82(15.5)$ & & $31(18.1)$ & $93(16.7)$ & \\
\hline With friends & $26(34.7)$ & $172(26.3)$ & & $48(24.3)$ & $150(28.3)$ & & 34 (19.9) & $164(29.4)$ & \\
\hline $\begin{array}{l}\text { Health related } \\
\text { majors }(\mathrm{n}, \%)\end{array}$ & $54(72)$ & $406(62.2)$ & $0.1^{\mathrm{b}}$ & $128(64.6)$ & $332(62.6)$ & $0.67^{\mathrm{b}}$ & $95(55.6)$ & $365(65.5)$ & $\begin{array}{l}0.02^{\mathrm{b}} \\
0.66(0.46-0.93)\end{array}$ \\
\hline $\begin{array}{l}\text { Level of educa- } \\
\text { tion (n, \%) }\end{array}$ & & & $0.07^{\mathrm{c}}$ & & & $0.02^{\mathrm{c}}$ & & & $0.82^{\mathrm{c}}$ \\
\hline 1st year & $7(9.3)$ & $106(16.2)$ & & 37 (18.7) & $76(14.3)$ & & $25(14.6)$ & $88(15.8)$ & \\
\hline 2nd year & $39(52.1)$ & $263(40.3)$ & & 83 (41.9) & 219 (41.3) & & $67(39.2)$ & $235(42.2)$ & \\
\hline 3rd year & $13(17.3)$ & $173(26.5)$ & & $57(28.8)$ & $129(24.3)$ & & $47(27.5)$ & $139(25)$ & \\
\hline $\begin{array}{l}\text { 4th year (final } \\
\text { year) }\end{array}$ & $16(21.3)$ & $111(17)$ & & $21(10.6)$ & $106(20.1)$ & & 32 (18.7) & 95 (17) & \\
\hline $\begin{array}{l}\text { Poor COVID- } \\
19 \text { knowledge }\end{array}$ & $61(81.3)$ & $289(44.3)$ & $\begin{array}{l}<0.001^{\mathrm{b}} \\
5.49(3.01- \\
10.01)\end{array}$ & $121(61.1)$ & $229(43.2)$ & $\begin{array}{l}<0.001^{\mathrm{b}} \\
2.06(1.48-2.88)\end{array}$ & $97(56.7)$ & $253(45.4)$ & $\begin{array}{l}0.01^{\mathrm{b}} \\
1.56(1.12-2.23)\end{array}$ \\
\hline
\end{tabular}

${ }^{a}$ t-test

${ }^{\mathrm{b}}$ Chi-squared test

${ }^{c}$ Chi-squared test for trend

*Grandparents, parents, uncles, and/or aunties

most of them (93.2\%) washed their masks after each use or after every day of use. There were still some participants (6.8\%) who washed their masks after some days of use but less than a week or after every week of use. It is documented that a well-washed cloth mask can be as protective as a medical mask [25]. This makes proper washing of face mask a key requirement in ensuring the effectiveness of face mask $[24,25]$. It is suggested that cloth mask should be washed appropriately in hot water and soap everyday [24, 25]. Although we did not document the methods of washing cloth masks, a number of our participants did not wash their cloth masks at least every day indicating the need to educate the community regarding the correct washing of cloth masks after use.

We found a significantly positive correlation between COVID-19 knowledge and all three components of KAP of face mask use. The findings of our study are in line with a previous study that showed an association between poor COVID-19 knowledge and knowledge of face mask use [26]. This study also showed that the participants' high knowledge of COVID-19 translated into good and safe practices, during the COVID-19 pandemic, which suggested a positive association between COVID-19 knowledge and correct implementation of preventive measures including face mask use [26]. Considering this, to promote correct use of face masks in the community, it is important to continue to educate the community regarding COVID-19 disease with an emphasis on the route of transmission and how correct use of face mask can stop the spread. We also found a significant association between face mask use attitudes and level of education. Although a study conducted in China found no association between levels of education and positive attitude among study participants [2], levels of education have been found to be associated with attitudes towards COVID-19 
and associated preventive measures in studies conducted in Saudi Arabia and Bangladesh [8, 26]. Interestingly, we found that studying health-related majors was statistically associated with good practice scores, but not positive attitudes towards face mask use. Given attitude comprises anything a person may hold in mind [27], like non-health-related students, some health-related students in our study may not really acknowledge the importance of using face mask as a preventive measure demonstrated by one-third $(27.2 \%)$ of all our study participants had negative attitudes towards face mask use. However, more health-related students had good practice scores compared with non-health students which could be due to their trainings in nursing and pharmacy. It is believed that their obligations and responsibilities to contribute to the control of this pandemic as future health professionals encourage them to have proactive practices during this pandemic [28]. We also found that poor practice of face mask use was significantly associated with male gender and living in rural area in our study participants. Our findings are in accordance with the findings of a study conducted in other countries including China, Saudi Arabia, and Bangladesh $[2,8,26]$. In light of this, it is important to enforce the mass masking policy among young men and those living in rural areas.

Our study has some limitations. First, this was a singlecenter study and thus, may not be a good representative of all Vietnamese university students. Nevertheless, our study was conducted on $81 \%$ of all enrolled students of one of the largest multidisciplinary universities in Vietnam. Second, given COVID-19 is a novel disease, there was no internationally validated questionnaire developed to measure KAP towards the use of face mask and other preventive measures. However, we developed our own questionnaire based on the recommendations of the WHO and Vietnam Ministry of Health $[13,14]$. To the best of our knowledge, like us, most similar published studies used questionnaires that were developed based on the guidelines of the WHO and their corresponding governments. Despite the limitations, this study provided valuable knowledge about KAP towards face mask use of university students-one of the prominent groups in Vietnam, which would be essential in improving the prevention and control of COVID-19 in Vietnam and comparable countries.

\section{Conclusion}

The levels of KAP towards face mask use among Vietnamese university students are high. It is important to continue to enforce the mass masking policy, as a means of preventing the spread of the virus together with the ongoing community education regarding COVID-19 disease with an emphasis on the route of transmission and how correct face mask use can stop the spread. To ensure the effectiveness of face mask use, there is an urgent need to educate the community regarding the correct reprocessing of cloth masks after use.

Supplementary Information The online version contains supplementary material available at https://doi.org/10.1007/s10900-021-00981-6.

Author Contributions MCD conceived the study. MCD and HTN developed the protocol. HTN collected data. MCD and BTD analyzed data. MCD, HTN, and BTD interpreted the findings and wrote the paper. All authors reviewed the paper for important intellectual content.

Data Availability All data and related material are included in the manuscript and Online Appendix.

\section{Declarations}

Conflict of interest The authors declare that they have no conflict of interest.

Ethical Approval The study procedure was performed in accordance with the ethical principles of the Declaration of Helsinki and was approved by the University Ethics Committee (Reference No. 09/TTKDD). Inform consent was obtained from all study participants.

\section{References}

1. Ruan, S. (2020). Likelihood of survival of coronavirus disease 2019. The Lancet Infectious Diseases, 20(6), 630-631.

2. Peng, Y., et al. (2020). A cross-sectional survey of knowledge, attitude and practice associated with COVID-19 among undergraduate students in China. BMC Public Health, 20(1), 1292.

3. Van Nguyen, Q., Cao, D. A., \& Nghiem, S. H. (2021). Spread of COVID-19 and policy responses in Vietnam: An overview. International Journal of Infectious Diseases: IJID: Official Publication of the International Society for Infectious Diseases, 103, 157-161.

4. Vietnam Ministry of Health. (2021). COVID-19 portal [in Vietnamese]. Retrieved February 17, 2021 from https://ncov.moh.gov. $\mathrm{vn} /$.

5. Van Tan, L. (2021). COVID-19 control in Vietnam. Nature Immunology, 22(3), 261-261.

6. Nguyen, N. P. T., et al. (2020). Preventive behavior of Vietnamese people in response to the COVID-19 pandemic. PLOS ONE, 15(9), e0238830.

7. Duong, M.C. (2020) Mass masking and Vietnam's COVID-19 success. Retrieved March 5, 2021 from https://www.eastasiaforum. org/2020/10/23/mass-masking-and-vietnams-covid-19-success/.

8. Ferdous, M. Z., et al. (2020). Knowledge, attitude, and practice regarding COVID-19 outbreak in Bangladesh: An online-based cross-sectional study. PLOS ONE, 15(10), e0239254.

9. Feng, S., et al. (2020). Rational use of face masks in the COVID19 pandemic. The Lancet Respiratory Medicine, 8(5), 434-436.

10. Greenhalgh, T., et al. (2020). Face masks for the public during the covid-19 crisis. BMJ, $369, \mathrm{~m} 1435$.

11. IndexMundi. (2019). Vietnam age structure. Retrieved February 19, 2021 from https://www.indexmundi.com/vietnam/age_struc ture.html.

12. Azlan, A. A., et al. (2020). Public knowledge, attitudes and practices towards COVID-19: A cross-sectional study in Malaysia. PLOS ONE, 15(5), e0233668. 
13. World Health Organization. (2020). Coronavirus disease (COVID19) advice for the public. Retrieved June 26, 2020 from https:// www.who.int/emergencies/diseases/novel-coronavirus-2019/ advice-for-public.

14. Ministry of Health. (2020) The ministry of health recommends " $5 K$ " to live safely during the COVID-19 pandemic [in Vietnamese]. Retrieved June 26, 2020 from https://ncov.moh.gov.vn/web/ guest/-/bo-y-te-khuyen-cao-5k-chung-song-an-toan-voi-dich-benh.

15. Worldometer. (2020) Covid-19 coronavirus pandemic. Retrieved March 1, 2021 from https://www.worldometers.info/coronavirus/.

16. Manikandan, N. (2020). Are social distancing, hand washing and wearing masks appropriate measures to mitigate transmission of COVID-19? Vacunas (English Edition), 21(2), 136-137.

17. Chiu, N.-C., et al. (2020). Impact of wearing masks, hand hygiene, and social distancing on influenza, enterovirus, and all-cause pneumonia during the coronavirus pandemic: Retrospective national epidemiological surveillance study. Journal of Medical Internet Research, 22(8), e21257-e21257.

18. Duong, D. M., Le, V. T., \& Ha, B. T. T. (2020). Controlling the COVID-19 pandemic in Vietnam: Lessons from a limited resource country. Asia-Pacific Journal of Public Health, 32(4), 161-162.

19. Duong, M.C. (2020). Mass masking and Vietnam's COVID-19 success. Retrieved March 5, 2021 from https://www.eastasiafo rum.org/2020/10/23/mass-masking-and-vietnams-covid-19-succe ss/.

20. Esposito, S., et al. (2020). Universal use of face masks for success against COVID-19: Evidence and implications for prevention policies. European Respiratory Journal, 55(6), 2001260.

21. Van Nhu, H., et al. (2020). Knowledge, attitudes, and practices of the Vietnamese as key factors in controlling COVID-19. Journal of Community Health, 45(6), 1263-1269.
22. Huong, L. T. T., et al. (2020). Reported handwashing practices of Vietnamese people during the COVID-19 pandemic and associated factors: A 2020 online survey. AIMS Public Health, 7(3), 650-663.

23. Zhong, B.-L., et al. (2020). Knowledge, attitudes, and practices towards COVID-19 among Chinese residents during the rapid rise period of the COVID-19 outbreak: A quick online cross-sectional survey. International Journal of Biological Sciences, 16(10), $1745-1752$.

24. MacIntyre, R. (2020). Cloth masks should be washed appropriately everyday. Retrieved March 5, 2021 from https://blogs.bmj. com/bmj/2020/09/29/raina-macintyre-cloth-masks-should-bewashed-appropriately-everyday/.

25. MacIntyre, C. R., et al. (2020). Contamination and washing of cloth masks and risk of infection among hospital health workers in Vietnam: A post hoc analysis of a randomised controlled trial. British Medical Journal Open, 10(9), e042045.

26. Al-Hanawi, M. K., et al. (2020). Knowledge, attitude and practice toward COVID-19 among the public in the kingdom of saudi arabia: A cross-sectional study. Frontiers in Public Health, 8 , 217-217.

27. Bohner, G., \& Dickel, N. (2011). Attitudes and attitude change. Annual Review of Psychology, 62, 391-417.

28. Heung, Y. Y. J., et al. (2005). Severe acute respiratory syndrome outbreak promotes a strong sense of professional identity among nursing students. Nurse Education Today, 25(2), 112-118.

Publisher's Note Springer Nature remains neutral with regard to jurisdictional claims in published maps and institutional affiliations.

\section{Authors and Affiliations}

\section{Minh Cuong Duong ${ }^{1}\left({ }^{1} \cdot\right.$ Hong Trang Nguyen ${ }^{2} \cdot$ Bich Thuy Duong ${ }^{3}$}

Hong Trang Nguyen

trang.nguyenhong@phenikaa-uni.edu.vn

Bich Thuy Duong

duongthuyicu@gmail.com

1 School of Population Health, University of New South

Wales, Samuels Building, F25, Samuel Terry Ave,

Kensington, NSW 2033, Australia
2 Faculty of Nursing, Phenikaa University, Yen Nghia ward, Ha Dong District, Hanoi 100000, Vietnam

3 Hospital for Tropical Diseases, Ho Chi Minh City 700000, Vietnam 